PROCEEDINGS OF THE

AMERICAN MATHEMATICAL SOCIETY

Volume 138, Number 10, October 2010, Pages 3585-3589

S 0002-9939(10)10358-X

Article electronically published on April 13, 2010

\title{
DOUBLING MEASURES WITH DOUBLING CONTINUOUS PART
}

\author{
MAN-LI LOU AND MIN WU
}

(Communicated by Michael T. Lacey)

\begin{abstract}
We prove that every compact subset of $\mathbb{R}^{d}$ of positive Lebesgue measure carries a doubling measure which is not purely atomic. Also, we prove that for every compact and nowhere dense subset $E$ of $\mathbb{R}^{d}$ without isolated points and for every doubling measure $\mu$ on $E$ there is a countable set $F$ with $E \cap F=\emptyset$ and a doubling measure $\nu$ on $E \cup F$ such that $\left.\nu\right|_{E}=\mu$. This shows that there are many doubling measures whose continuous part is doubling.
\end{abstract}

\section{Introduction AND MAIN RESUlts}

Doubling measure is an interesting topic in geometric measure theory. Many classic results, such as the Vitali covering lemma and the Lebesgue density theorem, can be extended from Lebesgue measure to doubling measure in Euclidean space. Doubling measures also arise in a natural way in various problems of analysis (see Stein [5]).

One can talk about doubling measure on any metric space. Let $(X, \rho)$ be a metric space. Denote by $B(x, r)$ the closed ball with center $x$ and radius $r$. A Borel measure $\mu$ on $X$ is doubling if there exists a constant $\lambda>1$ such that

$$
0<\mu(B(x, 2 r)) \leq \lambda \mu(B(x, r))<\infty
$$

for all $x \in X$ and $r>0$.

It is not hard to see that if a metric space $(X, \rho)$ carries a doubling measure, then there is a constant $N$ such that each $B(x, 2 r)$ in $X$ contains at most $N$ points with mutual distance at least $r$. A metric space with this property is called a doubling space. Conversely, $\mathrm{Vol}^{\prime}$ berg and Konyagin [6] proved that every doubling and compact metric space carries a doubling measure. Moreover, a somewhat more precise assertion holds for the case of $X \subset \mathbb{R}^{d}$.

Theorem (Vol'berg and Konyagin [6, Theorem 2]). Let $X$ be a compact subset of $\mathbb{R}^{d}$. Then $X$ carries a doubling measure $\nu$ such that

$$
\nu(B(x, k r)) \leq C k^{d} \nu(B(x, r))
$$

for all $x \in X, r>0, k \geq 1$, where the constant $C$ is independent of $k, x$, and $r$.

Received by the editors November 16, 2009 and, in revised form, December 30, 2009.

2010 Mathematics Subject Classification. Primary 28C15.

Key words and phrases. doubling measure, purely atomic, continuous part.

This work was supported by National Natural Science Foundation of China (Grants No. 10571063, 10631040).

(C)2010 American Mathematical Society Reverts to public domain 28 years from publication 
Luukkainen and Saksman [3] proved a further result that a complete metric space $X$ carries a doubling measure if and only if $X$ is doubling. Saksman [4 obtained some interesting results on the nonexistence of doubling measures.

We say that a Borel measure $\mu$ on a metric space $X$ is purely atomic if $\mu$ is supported by a countable subset of $X$. The following facts (see [1, 8]) are well known: (1) For each $\alpha \in[0,1]$ there exists a compact set $X \subset \mathbb{R}^{1}$ of Hausdorff dimension $\alpha$ such that all doubling measures on $X$ are purely atomic. (2) There exists a compact set $X \subset \mathbb{R}^{1}$ of positive length which carries purely atomic doubling measures. (3) There is a compact set $X$ in which isolated points are dense such that no doubling measures on $X$ are purely atomic. Lou, Wen and Wu 2 further asked the following question: Is there a compact subset $X$ of $[0,1]$ of positive Lebesgue measure on which all doubling measures are purely atomic? The first main result of this paper gives a negative answer to this question.

Theorem 1. Let $X$ be a compact subset of $\mathbb{R}^{d}$ with positive Lebesgue measure. Then $X$ carries a doubling measure which is not purely atomic.

Let $X$ be a metric space and $\mu$ a Borel measure on $X$. Write $X=E \cup F$, where $E$ denotes the set of accumulation points of $X$ and $F$ the set of isolated points of $X$. Kaufman and $\mathrm{Wu}\left[1\right.$ asked: do there exist $X$ compact in $\mathbb{R}^{1}$, a doubling measure $\mu$ on $X$, such that $F$ is dense in $X$ and such that the restriction $\left.\mu\right|_{E}$ is also a doubling measure on $E$ ? We say that $\left.\mu\right|_{E}$ is the continuous part of $\mu$. Recently, Wang and Wen [7, Theorem 3] answered this question positively by an example. The second main result of this paper completely answers this question.

Theorem 2. Let $E$ be a compact and nowhere dense subset of $\mathbb{R}^{d}$ without isolated points. Let $\mu$ be a doubling measure on $E$. Then there is a countable set $F$ with $E \cap F=\emptyset$ and a doubling measure $\nu$ on $X:=E \cup F$ such that

(i) $E$ is the set of accumulation points of $X$;

(ii) $F$ is dense in $X$;

(iii) $\left.\nu\right|_{E}=\mu$.

\section{Proofs OF MAIN RESUlTS}

Proof of Theorem 1, Let $X$ be a compact subset of $\mathbb{R}^{d}$ with Lebesgue measure $\mathcal{L}(X)>0$. We shall construct a doubling measure on $X$ which is not purely atomic. Without loss of generality suppose that

$$
X \subset[0,1)^{d} \text { and }|X| \leq 1,
$$

where $|X|$ denotes the diameter of $X$. According to [6, Theorem 2], $X$ carries a doubling probability measure $\nu$ with

$$
\nu(B(x, k r)) \leq C k^{d} \nu(B(x, r))
$$

for all $x \in X, r>0, k \geq 1$, where $C$ is independent of $k, x$, and $r$. Taking $k=r^{-1}$ in the above inequality, we get

$$
\nu(B(x, r)) \geq C^{-1} r^{d} .
$$

If the measure $\nu$ is not purely atomic, there is nothing to prove. Thus suppose that $\nu$ is purely atomic. Write $X=E \cup F$, where $E$ is the set of accumulation points 
of $X$ and $F$ is the set of isolated points of $X$. Then $\nu(E)=0, \mathcal{L}(E)=\mathcal{L}(X)$, and $\bar{F}=X$. For every integer $n \geq 1$ let

$$
\begin{aligned}
\mathscr{I}_{n}=\left\{\left[i_{1} 2^{-n},\left(i_{1}+1\right) 2^{-n}\right) \times \cdots \times\left[i_{d} 2^{-n},\left(i_{d}+1\right) 2^{-n}\right):\right. & \\
i_{j} & \left.\in\left[0,2^{n}\right) \cap \mathbb{Z}, \quad 1 \leq j \leq d\right\}
\end{aligned}
$$

be a partition of $[0,1)^{d}$. For every cube $I$ in $\mathscr{I}_{n}$ which meets $E$ take a point $x_{I} \in I \cap E$ and write

$$
D_{n}=\left\{x_{I}: I \in \mathscr{I}_{n}, I \cap E \neq \emptyset\right\} .
$$

Then the cardinality $\sharp D_{n} \geq 2^{\text {nd }} \mathcal{L}(X)$. Now define

$$
\mu_{n}=\frac{1}{2} \nu+\frac{1}{2 \sharp D_{n}} \sum_{x \in D_{n}} \delta_{x},
$$

where $\delta_{x}$ is the Dirac measure supported on $x$. One easily has $\mu_{n}(E)=\frac{1}{2}$ and

$$
\nu(B(x, r)) \leq 2 \mu_{n}(B(x, r)) .
$$

Let $\mu$ be a weak* limit measure of the sequence $\left\{\mu_{n}\right\}$. For convenience suppose that $\left\{\mu_{n}\right\}$ converges to $\mu$ in the weak* sense. We will show that $\mu$ is the desired measure.

It is clear that $\mu$ is supported by $X$ with $\mu(E) \geq \frac{1}{2}$, and so $\mu$ is not purely atomic. We only need to show that $\mu$ is doubling on $X$. Let $x \in X$ and $0<r \leq 1$. By the weak* convergence,

$$
\mu(B(x, 3 r)) \leq \frac{1}{2} \nu(B(x, 4 r))+\liminf _{n \rightarrow \infty} \frac{1}{2 \sharp D_{n}} \sum_{y \in D_{n}} \delta_{y}(B(x, 4 r)) .
$$

Let $\mathcal{C}_{n}$ be the family of cubes in $\mathscr{I}_{n}$ meeting $B(x, 4 r)$. Then

$$
\sum_{y \in D_{n}} \delta_{y}(B(x, 4 r)) \leq \sharp \mathcal{C}_{n} \leq 2^{n d} \mathcal{L}\left(B\left(x, 4 r+2^{-n} \sqrt{d}\right)\right),
$$

which, combined with the inequality $\sharp D_{n} \geq 2^{\text {nd }} \mathcal{L}(X)$, yields

$$
\liminf _{n \rightarrow \infty} \frac{1}{2 \sharp D_{n}} \sum_{y \in D_{n}} \delta_{y}(B(x, 4 r)) \leq \liminf _{n \rightarrow \infty} \frac{\mathcal{L}\left(B\left(x, 4 r+2^{-n} \sqrt{d}\right)\right)}{2 \mathcal{L}(X)}=C_{1} r^{d} .
$$

Now it follows from (2) and (4) and the doubling property of $\nu$ that

$$
\mu(B(x, 3 r)) \leq \frac{1}{2} \nu(B(x, 4 r))+C_{1} r^{d} \leq C_{2} \nu(B(x, r)) .
$$

This together with (3) gives $\mu(B(x, 3 r)) \leq C_{3} \mu\left(B\left(x, \frac{3 r}{2}\right)\right)$, where the constants $C_{1}$, $C_{2}$, and $C_{3}$ are independent of $x$ and $r$. This proves that $\mu$ is doubling.

Proof of Theorem 2. The proof will go as follows: We first construct an appropriate countable set $F=\left\{b_{i}\right\}_{i \geq 1}$ such that $F \cap E=\emptyset$. For convenience we write

$$
r_{i}=\operatorname{dist}\left(b_{i}, E\right) \quad \text { and } \quad m_{i}=\mu\left(B\left(b_{i}, \frac{5}{2} r_{i}\right)\right) \text {. }
$$

Then we define

$$
X=E \cup F \quad \text { and } \quad \nu=\mu+\sum_{b_{i} \in F} m_{i} \cdot \delta_{b_{i}},
$$

where $\delta_{b_{i}}$ is the Dirac measure supported on $b_{i}$. Finally we show that the statements (i), (ii) and (iii) are true and that the measure $\nu$ is doubling on $X$. 
Let $\left\{a_{i}\right\}_{i \geq 1}$ be a dense subset of $E$. We inductively construct the set $F$ as follows: Choose $b_{1} \notin E$ with $\operatorname{dist}\left(a_{1}, b_{1}\right) \leq 2^{-1}$. Suppose that $b_{1}, b_{2}, \ldots, b_{j}$ have been selected and choose $b_{j+1} \notin E$ such that

$$
\begin{gathered}
\operatorname{dist}\left(a_{j+1}, b_{j+1}\right) \leq 2^{-j-1}, \\
r_{j+1} \leq r_{j} / 24, \\
m_{j+1} \leq 2^{-j-1+i} m_{i} \text { for all } 1 \leq i \leq j .
\end{gathered}
$$

Since $E$ is compact and nowhere dense and $\mu$ is doubling on $E$, there exists a point $b_{j+1}$ satisfying the conditions (6), (17) and (8). Clearly $F \cap E=\emptyset$. By the construction, we have $r_{j} \rightarrow 0, m_{j} \rightarrow 0$, and $\operatorname{dist}\left(a_{j}, b_{j}\right) \rightarrow 0$, as $j \rightarrow \infty$.

Now let $X$ and $\nu$ be defined as in (5). It is clear that the statements (i), (ii), and (iii) hold. To complete the proof, it suffices to show that $\nu$ is doubling on $X$. For this, we need to estimate $\nu(B(x, 2 r)) / \nu(B(x, r))$ for $x \in X$. Write

$$
k=\min \left\{i: b_{i} \in B(x, 2 r)\right\} .
$$

According to (8), we know that

$$
\nu(B(x, 2 r)) \leq \mu(B(x, 2 r))+\sum_{i \geq k} m_{i} \leq \mu(B(x, 2 r))+2 m_{k} .
$$

Let $\lambda$ denote the doubling constant of $\mu$. There are two cases to consider.

Case I: $x \in E$. Then $b_{k} \in B(x, 2 r)$, and so $r_{k} \leq 2 r$. Thus we have

$$
\begin{aligned}
\frac{\nu(B(x, 2 r))}{\nu(B(x, r))} \leq \frac{\mu(}{} & (x, 2 r))+2 m_{k} \\
\mu(B(x, r)) & \\
& \leq \frac{\mu(B(x, 2 r))+2 \mu\left(B\left(x, 2 r+\frac{5}{2} r_{k}\right)\right)}{\mu(B(x, r))} \leq \frac{3 \mu(B(x, 7 r))}{\mu(B(x, r))} \leq 3 \lambda^{3} .
\end{aligned}
$$

Case II: $x=b_{j} \in F$. For all $b_{i} \in F$, take $b_{i}^{\prime} \in E$ with $\operatorname{dist}\left(b_{i}, b_{i}^{\prime}\right)=\operatorname{dist}\left(b_{i}, E\right)$. There are two subcases to consider.

Case II-i: $x=b_{j} \in F$ and $k<j$. Since $2 r \geq \operatorname{dist}\left(b_{j}, b_{k}\right) \geq r_{k}-r_{j}$ and $r_{k} \geq 24 r_{j}$ (by (7)), we have

$$
\frac{2 r+r_{j}+\frac{5}{2} r_{k}}{r-r_{j}} \leq \frac{\frac{7}{2} r_{k}}{\frac{1}{2} r_{k}-\frac{3}{2} r_{j}} \leq 8
$$

Therefore,

$$
\begin{aligned}
& \frac{\nu\left(B\left(b_{j}, 2 r\right)\right)}{\nu\left(B\left(b_{j}, r\right)\right)} \leq \frac{\mu\left(B\left(b_{j}^{\prime}, 2 r+r_{j}\right)\right)+2 m_{k}}{\mu\left(B\left(b_{j}^{\prime}, r-r_{j}\right)\right)} \\
& \leq \frac{\mu\left(B\left(b_{j}^{\prime}, 2 r+r_{j}\right)\right)+2 \mu\left(B\left(b_{j}^{\prime}, 2 r+r_{j}+\frac{5}{2} r_{k}\right)\right)}{\mu\left(B\left(b_{j}^{\prime}, r-r_{j}\right)\right)} \leq 3 \lambda^{3} .
\end{aligned}
$$

Case II-ii: $x=b_{j} \in F$ and $k=j$. Then

$$
\frac{\nu\left(B\left(b_{j}, 2 r\right)\right)}{\nu\left(B\left(b_{j}, r\right)\right)} \leq \frac{\mu\left(B\left(b_{j}, 2 r\right)\right)+2 m_{j}}{\mu\left(B\left(b_{j}, r\right)\right)+m_{j}}=\frac{\mu\left(B\left(b_{j}, 2 r\right)\right)+2 \mu\left(B\left(b_{j}, \frac{5}{2} r_{j}\right)\right)}{\mu\left(B\left(b_{j}, r\right)\right)+\mu\left(B\left(b_{j}, \frac{5}{2} r_{j}\right)\right)} .
$$

If $r \leq \frac{5}{2} r_{j}$, we have

$$
\frac{\nu\left(B\left(b_{j}, 2 r\right)\right)}{\nu\left(B\left(b_{j}, r\right)\right)} \leq \frac{\mu\left(B\left(b_{j}^{\prime}, 6 r_{j}\right)\right)}{\mu\left(B\left(b_{j}^{\prime}, \frac{3}{2} r_{j}\right)\right)}+2 \leq \lambda^{2}+2 \leq 3 \lambda^{3} .
$$


Otherwise $r>\frac{5}{2} r_{j}$. Then

$$
\frac{2 r+r_{j}}{r-r_{j}} \leq 4
$$

Therefore,

$$
\frac{\nu\left(B\left(b_{j}, 2 r\right)\right)}{\nu\left(B\left(b_{j}, r\right)\right)} \leq \frac{\mu\left(B\left(b_{j}^{\prime}, 2 r+r_{j}\right)\right)+2 \mu\left(B\left(b_{j}, \frac{5}{2} r_{j}\right)\right)}{\mu\left(B\left(b_{j}^{\prime}, r-r_{j}\right)\right)+\mu\left(B\left(b_{j}, \frac{5}{2} r_{j}\right)\right)} \leq \lambda^{2}+2 \leq 3 \lambda^{3} .
$$

\section{ACKNOWLEDGMENTS}

We are extremely grateful to Professor Shengyou Wen for reading the whole manuscript carefully and for his helpful suggestions. We also thank Professor Jihua Ma and Doctor Ying Xiong for useful discussions.

\section{REFERENCES}

[1] Robert Kaufman and Jang-Mei Wu. Two problems on doubling measures. Rev. Mat. Iberoamericana, 11(3):527-545, 1995. MR1363204(97k:28006)

[2] Manli Lou, Shengyou Wen, and Min Wu. Two examples on atomic doubling measures. J. Math. Anal. Appl., 333(2):1111-1118, 2007. MR2331717 (2008m:28008)

[3] Jouni Luukkainen and Eero Saksman. Every complete doubling metric space carries a doubling measure. Proc. Amer. Math. Soc., 126(2):531-534, 1998. MR.1443161 (99c:28009)

[4] Eero Saksman. Remarks on the nonexistence of doubling measures. Ann. Acad. Sci. Fenn. Math., 24(1):155-163, 1999. MR.1678044 (2000b:28006)

[5] Elias M. Stein. Harmonic analysis: Real-variable methods, orthogonality, and oscillatory integrals, with the assistance of Timothy S. Murphy, volume 43 of Princeton Mathematical Series. Monographs in Harmonic Analysis, III, Princeton University Press, Princeton, NJ, 1993. MR $1232192(95 \mathrm{c}: 42002)$

[6] A. L. Vol'berg and S. V. Konyagin. On measures with the doubling condition. Izv. Akad. Nauk SSSR Ser. Mat., 51(3):666-675, 1987. MR903629 (88i:28006)

[7] Xiaohua Wang and Shengyou Wen. Doubling measures on Cantor sets and their extensions. preprint.

[8] Jang-Mei Wu. Hausdorff dimension and doubling measures on metric spaces. Proc. Amer. Math. Soc., 126(5):1453-1459, 1998. MR 1443418 (99h:28016)

Department of Mathematics, South China University of Technology, Guangzhou, 510641, People's Republic of China

E-mail address: loumanli@126.com

Department of Mathematics, South China University of Technology, Guangzhou, 510641, People's Republic of China

E-mail address: wumin@scut.edu.cn 